\title{
Saddle solutions of nonlinear elliptic equations involving the $p$-Laplacian
}

\author{
Zhuoran Du, Zheng Zhou and Baishun Lai
}

\begin{abstract}
We prove the existence of saddle solutions of nonlinear elliptic equation involving the $p$-Laplacian

$$
-\Delta_{p} u=f(u) \text { in } R^{n}
$$

where $p>2, n:=2 m \geq 2 p$. We also get a pointwise estimate of the saddle solutions.

Mathematics Subject Classification (1991). 35J60, 35J70, 35J20, 34D20, 34C37.
\end{abstract}

Keywords. $p$-Laplacian, Saddle solutions, Variational methods.

\section{Introduction and main results}

In this paper we study saddle solutions of nonlinear elliptic problem involving the $p$-Laplacian

$$
-\Delta_{p} u:=-\operatorname{div}\left(|\nabla u|^{p-2} \nabla u\right)=f(u) \quad \text { in } R^{n} .
$$

where $p>2, n:=2 m \geq 2 p$. Nonlinearity $f(u)$ satisfies several conditions (1.9)(1.11) as stated at the end of this section.

In 1978 De Giorgi [12] made the following celebrated conjecture (De Giorgi conjecture): Let $u \in C^{2}\left(R^{n}\right)$ be a solution of

$$
-\Delta u=u-u^{3}, \quad|u| \leq 1, \quad u_{x_{n}}>0 \quad \text { in } R^{n} .
$$

Is it true that all the level sets of $u$ are hyperlanes, at least if $n \leq 8$ ?

In the last years there has been special interest on De Giorgi conjecture. Ghoussoub and Gui [15] first proved this conjecture for $n=2$, and Berestycki et al. [5] also got the same result for dimension $n=2$. Ambrosio and Cabré proved it for $n=3$ in [4]. The symmetry result in dimension $n=3$ was extended to all $C^{2}$ nonlinearities in [2]. Also for $n=4$ or $n=5$, this conjecture was proved by Ghoussoub and Gui [16] for a special class of solutions 
that satisfy an anti-symmetry condition. For $4 \leq n \leq 8$, assuming an additional limiting condition on $u$, Savin proved that this conjecture is also true [19]. Recently, del Pino et al. [13] have announced that the statement of the conjecture does not hold for $n \geq 9$, by constructing a counterexample. Danielli and Garofalo [11] and Valdinoci et al. [22] extended De Giorgi conjecture for $p=2$ to general case $p>1$.

One would wish to seek for an alternative way to that of [13]. By a result of Jerison and Monneau [17], the existence of a counter-example to the conjecture in $R^{n+1}$ would be established if one could prove the existence of a bounded, even with respect to each coordinate, global minimizer of (1.2) in $R^{n}$. On the other hand, Savin obtained a deep result in [19] that every global minimizer is an odd function of only one Euclidean variable for $n \leq 7$. In particular, an even function with respect to each coordinate can not be a global minimizer in $R^{n}$ whenever $n \leq 7$. The remaining question is whether a global minimizer of (1.2), even function with respect to each coordinate, exists in higher dimensions. A natural candidate is expected to be founded in the class of saddle solutions. For this aim, Cabré and Terra [6] studied the existence of saddle solutions of $-\Delta u=f(u)$ in every even dimension and instability of its saddle solutions in low even dimensions. Extending the Laplace operator to $p$-Laplace operator, we will consider the existence of saddle solutions of (1.1) in this paper.

Saddle solutions were first studied by Dang et al. [10] for equation $-\Delta u=$ $f(u)$ in $R^{2}$, with $f$ odd, bistable and $f(u) / u$ decreasing for $u \in(0,1)$. They proved the existence and uniqueness of a saddle solution. They also established monotonicity properties and the asymptotic behavior of the saddle solution. Schatzman [20] studied in detail its instability, which was already indicated in a partial result of [10]. Alama et al. [1] studied vector-valued saddle solutions in $R^{2}$. The article [3] concerns scalar saddle type solutions in $R^{2}$ changing sign on more nodal lines than $x_{1}= \pm x_{2}$. Recently, Cabré and Terra proved the existence of saddle solutions of bistable diffusion equations $-\Delta u=f(u)$ in all even dimensions. They also obtained the instability of saddle solutions in $R^{4}$ and $R^{6}$, see $[6,7]$.

Before introducing the notion of saddle solution, we give the weak solution notion.

Definition 1.1. We say that $u$ is a bounded weak solution of

$$
-\Delta_{p} u=f(u) \quad \text { in } R^{2 m}
$$

if $u \in W_{l o c}^{1, p}\left(R^{2 m}\right) \cap L^{\infty}\left(R^{2 m}\right)$, and

$$
\int_{R^{2 m}}\left[|\nabla u|^{p-2} \nabla u \cdot \nabla \eta-f(u) \eta\right] \mathrm{d} x=0, \quad \text { for all } \eta \in W_{0}^{1, p}\left(R^{2 m}\right) .
$$

Saddle solution notion is closely related to the Simons cone. For $n=2 m$, the Simons cone is defined by

$$
\mathcal{C}=\left\{x \in R^{2 m}: x_{1}^{2}+\cdots+x_{m}^{2}=x_{m+1}^{2}+\cdots+x_{2 m}^{2}\right\} .
$$


We define two radial variables $s$ and $t$ by

$$
s=\sqrt{x_{1}^{2}+\cdots+x_{m}^{2}} \geq 0, \quad t=\sqrt{x_{m+1}^{2}+\cdots+x_{2 m}^{2}} \geq 0 .
$$

Then the Simons cone is given by $\mathcal{C}=\{s=t\}$. It is easy to verify that $\mathcal{C}$ has zero mean curvature at every $x \in \mathcal{C} \backslash\{0\}$, in every even dimension $2 m \geq 2$. The following is the precise notion of saddle solution, see [6].

Definition 1.2. Let $f \in C^{1}(R)$ be odd. We say that $u$ is a saddle solution of (1.3), if $u$ is a bounded weak solution of (1.3) and

(1) $u$ depends only on the variables $s$ and $t$. We write $u=u(s, t)$;

(2) $u>0$ in $\mathcal{H}:=\{s>t\}$;

(3) $u(s, t)=-u(t, s)$ in $R^{2 m}$.

Condition (3) implies that every saddle solution vanishes on the Simons cone $\mathcal{C}=\partial \mathcal{H}=\{s=t\}$. Note also that saddle solutions are even with respect to each coordinate $x_{i}, 1 \leq i \leq 2 m$.

Equation (1.1) is the Euler-Lagrange equation associated to the energy functional

$$
J(v, \Omega)=\int_{\Omega}\left(\frac{|\nabla v|^{p}}{p}+F(v)\right) \mathrm{d} x
$$

where $F^{\prime}=-f$ and $\Omega \subset R^{n}$ is a bounded domain. From the energy $J$ we may give the definition of stability.

Definition 1.3. Suppose $f \in C^{1}(R)$.

(i) We say that a bounded $C^{1}$ function $u: R^{n} \rightarrow R$ is a global minimizer of (1.1) if

$$
J(u, \Omega) \leq J(u+\eta, \Omega)
$$

for every bounded domain $\Omega$ and every $C^{\infty}$ function $\eta$ with compact support in $\Omega$.

(ii) Suppose also $p>2$. We say that a bounded weak solution $u$ of (1.1) is stable if the second variation of energy with respect to compactly supported perturbations $\xi$ is nonnegative. That is, if

$$
\begin{aligned}
Q_{u}(\xi)= & \int_{R^{n}}\left(|\nabla u|^{p-2}|\nabla \xi|^{2}+(p-2)|\nabla u|^{p-4}|\nabla u \cdot \nabla \xi|^{2}\right. \\
& \left.-f^{\prime}(u) \xi^{2}\right) \mathrm{d} x \geq 0 \quad \text { for all } \xi \in C_{c}^{\infty}\left(R^{n}\right) .
\end{aligned}
$$

We say that $u$ is unstable if and only if $u$ is not stable.

Obviously, every global minimizer is a stable solution.

To state our main results in this paper, given a $C^{1}$ nonlinearity $f: R \rightarrow R$ and $M>0$, define

$$
F(u)=\int_{u}^{M} f
$$


We have that $F \in C^{2}(R)$ and $F^{\prime}=-f$. For $p>2$, we assume that, there exist $0<c<1<C, \widetilde{C}>0$ and some $w^{\star} \in(0, M)$ such that

$F$ is nonnegative in $R$. For any $w \in[0, M], c w^{p} \leq F(-M+w) \leq C w^{p}$

$$
\text { and } c w^{p} \leq F(M-w) \leq C w^{p}
$$

$f$ is odd in $R$, and $f$ is positive in $(0, M)$;

for any $w \in\left[0, w^{\star}\right), f(-M+w) \geq-\widetilde{C} w^{p-1}$ and $f(M-w) \leq \widetilde{C} w^{p-1}$.

Note that if conditions (1.9)-(1.11) hold, then $F$ is even in $R, F(M)=$ $F(-M)=0$ and $F>0$ in $(-M, M)$, as well as $f(0)=f( \pm M)=0$ is available. The potentials $F$ are of "double-well type". Our three assumptions (1.9)-(1.11) are satisfied for equation $-\Delta_{p} u=u\left(1-u^{2}\right)\left|1-u^{2}\right|^{p-2}$. In this case, $M=1$ and $F(u)=\frac{\left|1-u^{2}\right|^{p}}{2 p}$, which is the typical double well for the $p$-Laplacian case, as considered, for instance in [22].

Our main results in this paper are as follows.

Theorem 1.4. Let $p>2$ and $f \in C^{1}(R)$ satisfy (1.9)-(1.11) for some constant $M>0$, where $F$ is defined by (1.8). Then for every even dimension $2 m \geq 2 p>4$, there exists a saddle solution $u$ of equation (1.3). In addition, $|u|<M$ in $R^{2 m}$, as well as the energy estimate

$$
J\left(u, B_{R}\right)=\int_{B_{R}}\left(\frac{|\nabla u|^{p}}{p}+F(u)\right) \mathrm{d} x \leq C R^{2 m-1} \quad \text { for all } R>1,
$$

where $C$ is a constant independent of $R$ and $B_{R}$ denotes the open ball of radius $R$ centered at 0 .

Especially, if $f$ also satisfies $\frac{f^{\prime}(w)}{f(w)} \leq \frac{p-1}{w}$ in $(0, M),{ }^{1}$ then the second variation of energy $Q_{u}(\xi)$ at $u$, as defined in (1.7), is nonnegative for all functions $\xi \in C^{1}\left(R^{2 m}\right)$ with compact support in $R^{2 m}$ and vanishing on the Simons cone $\mathcal{C}=\{s=t\}$.

We stress that the stability here is only valid for tests functions supported outside the boundary of the cone. We want to point out that (1.12) is also true for global minimizers, as shown by Caffarelli and Cordoba [8] when $p=2$, and by Petrosyan and Valdinoci [18] When $p>1$. Non global minimizers, in general, do not satisfy (1.12), as the zero function shows (see the proof of Theorem 1.4 for more details). So it is somewhat surprising that (1.12) holds for saddle solutions.

Theorem 1.5. Let $f \in C^{1}(R)$ satisfy (1.9) and (1.10), where $F$ is defined by (1.8). If $u$ is a bounded weak solution of $-\Delta_{p} u=f(u)$ in $R^{2 m}$ that vanishes on the Simons cone $\mathcal{C}=\{s=t\}$, then

$$
|u(x)| \leq\left|u_{0}(\operatorname{dist}(x, \mathcal{C}))\right|=\left|u_{0}\left(\frac{s-t}{\sqrt{2}}\right)\right| \quad \text { for all } x \in R^{2 m},
$$

\footnotetext{
${ }^{1}$ Inequality $\frac{f^{\prime}(w)}{f(w)} \leq \frac{p-1}{w}$ in $(0, M)$ implies that $\frac{f(w)}{w^{p-1}}$ is decreasing in $(0, M)$.
} 
where $u_{0}$ is the monotone solution of $-\left(\left|u^{\prime}\right|^{p-2} u^{\prime}\right)^{\prime}=f(u)$ in $R$ vanishing at 0 (see Lemma 2.5). In particular, this statement holds true for every saddle solution.

In addition, the function $u_{0}\left(\frac{s-t}{\sqrt{2}}\right)$ is a supersolution of $-\Delta_{p} u=f(u)$ in the set $\mathcal{H}=\{s>t\}$.

\section{Preliminaries}

This section contain several properties which will be used to prove Theorems 1.4 and 1.5, as well as the existence of solution to the corresponding one dimensional problem.

Caffarelli et al. in [9] studied a gradient bound for entire solutions of equation

$$
\operatorname{div}\left(\Phi^{\prime}\left(|\nabla u|^{2}\right) \nabla u\right)=F^{\prime}(u) .
$$

By assuming conditions on $\Phi \in C^{3}\left(R^{+}\right)$:

$$
\left\{\begin{aligned}
& c_{1}(a+|\sigma|)^{p-2} \leq \Phi^{\prime}\left(|\sigma|^{2}\right) \leq c_{2}(a+|\sigma|)^{p-2}, p>1, a \geq 0, c_{1}, c_{2}>0, \\
& c_{1}\left(a+|\sigma|^{p-2}\right)|\xi|^{2} \leq \sum_{i, j=1}^{n} a_{i j}(\sigma) \xi_{i} \xi_{j} \\
& \leq c_{2}\left(a+|\sigma|^{p-2}\right)|\xi|^{2}, \forall \sigma, \xi \in R^{n} \backslash\{0\},
\end{aligned}\right.
$$

where $a_{i j}(\sigma)=2 \Phi^{\prime \prime}\left(|\sigma|^{2}\right) \sigma_{i} \sigma_{j}+\Phi^{\prime}\left(|\sigma|^{2}\right) \delta_{i j}$, they obtained the following result.

Theorem 2.1. Let $F \in C^{2}(R)$ with $F \geq 0$, and suppose (2.2) holds and $u \in$ $W_{\text {loc }}^{1, p}\left(R^{n}\right) \cap L^{\infty}\left(R^{n}\right)$ is a weak solution to (2.1) in $R^{n}$, then, for every $x \in R^{n}$ we have

$$
2 \Phi^{\prime}\left(|\nabla u(x)|^{2}\right)|\nabla u(x)|^{2}-\Phi\left(|\nabla u(x)|^{2}\right) \leq 2 F(u(x)) .
$$

In particular, nonlinear elliptic equations (1.1) involving the $p$-Laplacian corresponds to the choice $\Phi(s)=\frac{2}{p} s^{\frac{p}{2}}$. In this case, (2.3) reads

$$
|\nabla u(x)|^{p} \leq \frac{p}{p-1} F(u(x)), \quad x \in R^{n} .
$$

In the same paper, three authors also got the following Liouville type result.

Theorem 2.2. Let $u$ be a weak solution of (2.1) and suppose that the assumptions of Theorem 2.1 are satisfied. We also require that if $F\left(u_{0}\right)=0$, then $F(u)=O\left(\left|u-u_{0}\right|^{p}\right)$ as $u \rightarrow u_{0}$, when $p \geq 2$.

If there exists $x_{0} \in R^{n}$ such that $F\left(u\left(x_{0}\right)\right)=0$ then $u \equiv$ const in $R^{n}$.

We now state a strong maximum principle proved by Vazquez [23].

Theorem 2.3. Let $\Omega$ be an open connected (not necessarily) bounded set in $R^{n}$ and suppose that $u \in C^{1}(\Omega), u \geq 0$ in $\Omega$, weakly satisfies

$$
-\Delta_{p} u+c u^{q}=g \geq 0 \quad \text { in } \Omega
$$

with $q \geq p-1, c \geq 0$ and $g \in L_{\text {loc }}^{\infty}$. If $u$ is not identically zero, then $u>0$ in $\Omega$. 
By Theorem 2.3, one can obtain the following lemma, see footnote 5 in $[21]$.

Lemma 2.4. Let $f \in C^{1}(R)$ satisfies (1.11). Function u weakly solves

$$
-\Delta_{p} u=f(u) \text { in } \Omega \text {, }
$$

where $\Omega$ is an connected open set in $R^{n}$. If $|u| \leq M$ and $|u| \not \equiv M$, then $|u|<M$.

In the rest of this section, we will get the existence of solution to nonlinear equation related to the $p$-Laplacian in dimension one.

Lemma 2.5. Let $F \in C^{2}(R)$. There exists a bounded function $u_{0} \in C^{2}(R)$ satisfying

$$
\left(\left|u_{0}^{\prime}\right|^{p-2} u_{0}^{\prime}\right)^{\prime}=F^{\prime}\left(u_{0}\right), \quad \text { and } \quad u_{0}^{\prime}>0 \quad \text { in } R
$$

if and only if there exist two real numbers $m_{1}<m_{2}$ for which $F$ satisfies

$$
F^{\prime}\left(m_{1}\right)=F^{\prime}\left(m_{2}\right)=0
$$

and

$$
F>F\left(m_{1}\right)=F\left(m_{2}\right) \quad \text { in }\left(m_{1}, m_{2}\right) .
$$

In such case we have $m_{1}=\lim _{\tau \rightarrow-\infty} u_{0}(\tau)$ and $m_{2}=\lim _{\tau \rightarrow \infty} u_{0}(\tau)$. Moreover, the solution $u_{0}=u_{0}(\tau)$ is unique up to translations of the independent variable $\tau$.

Adding a constant to $F$, assume that

$$
F\left(m_{1}\right)=F\left(m_{2}\right)=0,
$$

then we have that

$$
\frac{p-1}{p}\left|u_{0}^{\prime}\right|^{p}=F\left(u_{0}\right) \quad \text { in } R .
$$

Proof. We first prove the necessity. Since $u_{0} \in C^{2}(R)$, the equation may be written as

$$
(p-1)\left|u_{0}^{\prime}\right|^{p-2} u_{0}^{\prime \prime}=F^{\prime}\left(u_{0}\right) .
$$

Multiplying (2.9) by $u_{0}^{\prime}$ and integrating, we find that

$$
\frac{p-1}{p}\left|u_{0}^{\prime}\right|^{p}-F\left(u_{0}\right)=C \quad \text { in } R,
$$

where $C$ is a constant. Since $u_{0}$ has finite limit as $\tau \rightarrow \pm \infty$ we obtain

$$
\liminf _{\tau \rightarrow \pm \infty} u_{0}^{\prime}(\tau)=0
$$

whence $C$ is equal to $-F\left(m_{1}\right)$ and $-F\left(m_{2}\right)$, where $m_{1}=\lim _{\tau \rightarrow-\infty} u_{0}(\tau)$ and $m_{2}=\lim _{\tau \rightarrow \infty} u_{0}(\tau)$. In view of $F\left(u_{0}\right)=\frac{p-1}{p}\left|u_{0}^{\prime}\right|^{p}-C>-C$ and the image of $u_{0}$ is $\left(m_{1}, m_{2}\right)$, we infer (2.6). The equalities $F^{\prime}\left(m_{1}\right)=F^{\prime}\left(m_{2}\right)=0$ follow from the equation (2.9) and equality (2.10). 
Now we prove the sufficiency. We assume that there is a solution $u_{0}$ and try to solve it explicitly. Without loss of generality, we may assume $u_{0}(0)=m$, for some $m \in\left(m_{1}, m_{2}\right)$. We let $G:\left(m_{1}, m_{2}\right) \rightarrow R$ be the function

$$
G(u)=\int_{m}^{u} \frac{1}{\left(\frac{p}{p-1}\left[F(s)-F\left(m_{1}\right)\right]\right)^{\frac{1}{p}}} \mathrm{~d} s
$$

well defined thanks to (2.6). Then $G^{\prime}(u)>0, u \in\left(m_{1}, m_{2}\right)$.

Due to $u_{0}^{\prime}>0$ in $R$, from [11] we know that equality $\frac{p-1}{p}\left|u_{0}^{\prime}\right|^{p}-F\left(u_{0}\right) \equiv C$ holds. Clearly, $C=-F\left(m_{1}\right)$. Hence, we have

$$
\frac{u_{0}^{\prime}(\tau)}{\left(\frac{p}{p-1}\left[F\left(u_{0}\right)-F\left(m_{1}\right)\right]\right)^{\frac{1}{p}}}=1, \quad \tau \in R .
$$

Integrating (2.11) from 0 to $\tau$, we get

$$
G\left(u_{0}(\tau)\right)=\tau \quad \text { or } \quad u_{0}(\tau)=G^{-1}(\tau), \quad \tau \in R .
$$

Generally, the solution $u_{0} \in C^{1, \alpha}(R)$ only, however, since $u_{0}^{\prime}>0$ in $R$, the regularity of ode's guarantee that $u_{0} \in C^{2}(R)$. Therefore $u_{0}$ is a classical solution of $(2.5)$.

\section{Proof of Theorems 1.4 and 1.5}

We first use a variational method to prove Theorem 1.4, following the main ideas in [6].

Recalling the energy functional

$$
J(u, \Omega)=\int_{\Omega}\left(\frac{|\nabla u|^{p}}{p}+F(u)\right) \mathrm{d} x
$$

note that for functions $u$ depending only on $s$ and $t$, such as saddle solutions, the energy functional becomes

$$
J(u, \Omega)=a_{m} \int_{\Omega} s^{m-1} t^{m-1}\left(\frac{1}{p}\left(u_{s}^{2}+u_{t}^{2}\right)^{\frac{p}{2}}+F(u)\right) \mathrm{d} s \mathrm{~d} t,
$$

where $a_{m}$ is a positive constant depending on $m$. Here we have assumed that $\Omega \subset R^{2 m}$ is radially symmetric in the first $m$ variables and also in the last $m$ variables, and we have abused notation by identifying $\Omega$ with its projection in the $(s, t)$ variables. In these variables, Eq. (1.3) reads

$$
\begin{gathered}
-\left(u_{s}^{2}+u_{t}^{2}\right)^{\frac{p-2}{2}}\left(u_{s s}+u_{t t}\right)-(m-1)\left(u_{s}^{2}+u_{t}^{2}\right)^{\frac{p-2}{2}}\left(\frac{u_{s}}{s}+\frac{u_{t}}{t}\right) \\
-(p-2)\left(u_{s}^{2}+u_{t}^{2}\right)^{\frac{p-4}{2}}\left(u_{s}^{2} u_{s s}+2 u_{s} u_{t} u_{s t}+u_{t}^{2} u_{t t}\right) \\
=f(u), \quad s>0, \quad t>0 .
\end{gathered}
$$

To prove the existence of a saddle solution in $R^{2 m}=\left\{x=\left(x^{1}, x^{2}\right) \in R^{m} \times\right.$ $\left.R^{m}\right\}$, using a variational technique, we will construct a solution $u$ in $\mathcal{H}$ satisfying $u>0$ in $\mathcal{H}$ and $u=0$ on $\mathcal{C}=\partial \mathcal{H}$. Then since $f$ is odd, by odd reflection with respect to the cone $\mathcal{C}$ we obtain a saddle solution in the whole space. Now we prove Theorem 1.4 in detail. 
Proof. Let $B_{R}$ be the open ball in $R^{2 m}$ centered at the origin and of radius $R$. We set

$$
\mathcal{H}_{R}=\mathcal{H} \cap B_{R}=\left\{s>t \text { and }|x|^{2}=s^{2}+t^{2}<R^{2}\right\} .
$$

Note that $\partial \mathcal{H}_{R}=\left(\mathcal{C} \cap \bar{B}_{R}\right) \cup\left(\partial B_{R} \cap \mathcal{H}\right)$. We introduce the space

$$
\widetilde{W}_{0}^{1, p}\left(\mathcal{H}_{R}\right):=\left\{v \in W_{0}^{1, p}\left(\mathcal{H}_{R}\right): v=v(s, t) \text { a.e. }\right\}
$$

of $W_{0}^{1, p}$ functions in the bounded open set $\mathcal{H}_{R}$ which depends only on $s$ and $t$. It is easy to verify that $\widetilde{W}_{0}^{1, p}\left(\mathcal{H}_{R}\right)$ is a weakly closed subspace of $W_{0}^{1, p}\left(\mathcal{H}_{R}\right)$.

Consider the energy functional in $\mathcal{H}_{R}$

$$
J\left(v, \mathcal{H}_{R}\right)=\int_{\mathcal{H}_{R}}\left(\frac{|\nabla v|^{p}}{p}+F(v)\right) \mathrm{d} x, \quad \text { for } v \in \widetilde{W}_{0}^{1, p}\left(\mathcal{H}_{R}\right) .
$$

We may show the existence of a minimizer of the functional among functions in space $\widetilde{W}_{0}^{1, p}\left(\mathcal{H}_{R}\right)$. Indeed, recall that $F$ satisfying

$$
F \geq 0=F( \pm M) \text { in } R \text { and } F>0 \text { in }(-M, M) .
$$

Since $J$ is nonnegative, we can take a minimizing sequence $\left\{u_{R}^{k}\right\}, k=1,2, \ldots$ of $J$ in $\widetilde{W}_{0}^{1, p}\left(\mathcal{H}_{R}\right)$. With no loss of generality we may assume that $0 \leq u_{R}^{k} \leq M$. To see this, simply replace the minimizing sequence $\left\{u_{R}^{k}\right\}$ by the sequence $\left\{v_{R}^{k}\right\}$, defined by $v_{R}^{k}=\min \left\{\left|u_{R}^{k}\right|, M\right\} \in \widetilde{W}_{0}^{1, p}\left(\mathcal{H}_{R}\right)$, which is also a minimizing sequence. As a matter of fact, since $F$ is even, then sequence $\left\{\left|u_{R}^{k}\right|\right\}$ is a minimizing, also noting that $F \geq F(M)$ we can conclude that $\left\{v_{R}^{k}\right\}$ is also minimizing.

Since $F \geq 0$, we have

$$
\int_{\mathcal{H}_{R}}\left|\nabla u_{R}^{k}\right|^{p} \mathrm{~d} x \leq p J\left(u_{R}^{k}, \mathcal{H}_{R}\right) \leq C
$$

for some constant $C$. Hence there exists a subsequence (denoted again by $\left\{u_{R}^{k}\right\}$ ) such that $u_{R}^{k}$ converges weakly in $W_{0}^{1, p}\left(\mathcal{H}_{R}\right)$ to a function $u_{R} \in \widetilde{W}_{0}^{1, p}\left(\mathcal{H}_{R}\right)$. Due to the weak convergence we get

$$
\int_{\mathcal{H}_{R}}\left|\nabla u_{R}\right|^{p} \mathrm{~d} x \leq \liminf _{k} \int_{\mathcal{H}_{R}}\left|\nabla u_{R}^{k}\right|^{p} \mathrm{~d} x .
$$

By Fatou's lemma, we also have

$$
\int_{\mathcal{H}_{R}} F\left(u_{R}\right) \mathrm{d} x \leq \liminf _{k} \int_{\mathcal{H}_{R}} F\left(u_{R}^{k}\right) \mathrm{d} x .
$$

So $u_{R}$ is a minimizing function of $J\left(\cdot, \mathcal{H}_{R}\right)$ in $\widetilde{W}_{0}^{1, p}\left(\mathcal{H}_{R}\right)$ and $0 \leq u_{R} \leq M$ in $\mathcal{H}_{R}$.

Next, we can consider perturbations $u_{R}+\xi$ of $u_{R}$, with $\xi$ depending only on $s$ and $t$, and with $\xi$ having compact support in $\mathcal{H}_{R} \cap\{t>0\}=B_{R} \cap$ $\{0<t<s\}$. In particular, $\xi$ vanishes in a neighborhood of $\{t=0\}$. Since Eq. (3.2) in the $(s, t)$ variables is the first variation of $J\left(\cdot, \mathcal{H}_{R}\right)$, which has the form in (3.1) on $\widetilde{W}_{0}^{1, p}$ functions, and condition $p>2$ shows that the equation 
is not singular away from $\{s=0\}$ and $\{t=0\}$, we deduce that $u_{R}$ is a weak solution of (3.2) in $\mathcal{H}_{R} \cap\{t>0\}$. That is, $u_{R}$ weakly satisfies

$$
-\Delta_{p} u_{R}=f\left(u_{R}\right) \quad \text { in } \mathcal{H}_{R} \cap\{t>0\} .
$$

Namely

$$
\int_{\mathcal{H}_{R}}\left[\left|\nabla u_{R}\right|^{p-2} \nabla u_{R} \cdot \nabla \eta-f\left(u_{R}\right) \eta\right] \mathrm{d} x=0, \quad \text { for all } \eta \in W_{0}^{1, p}\left(\mathcal{H}_{R} \cap\{t>0\}\right) .
$$

We now prove that $u_{R}$ is also a weak solution in all of $\mathcal{H}_{R}$, that is, also across $\{t=0\}$. To verify this, we let $\eta=v \xi_{\varepsilon}$ in $(3.3)$, where $v \in C_{c}^{\infty}\left(\mathcal{H}_{R}\right)$ and $\xi_{\varepsilon}$ be a smooth function of $t$ alone being identically 0 in $\left\{t<\frac{\varepsilon}{2}\right\}$ and identically 1 in $\{t>\varepsilon\}$. Hence, (3.3) becomes

$$
\begin{aligned}
& \int_{\mathcal{H}_{R}} \xi_{\varepsilon}\left|\nabla u_{R}\right|^{p-2} \nabla u_{R} \cdot \nabla v \mathrm{~d} x+\int_{\mathcal{H}_{R} \cap\{t<\varepsilon\}} v\left|\nabla u_{R}\right|^{p-2} \nabla u_{R} \cdot \nabla \xi_{\varepsilon} \mathrm{d} x \\
& \quad=\int_{\mathcal{H}_{R}} f\left(u_{R}\right) v \xi_{\varepsilon} \mathrm{d} x
\end{aligned}
$$

We need to show that the second integral on the left hand side of (3.4) goes to zero as $\varepsilon \rightarrow 0$. Indeed, by Hölder inequality, we have

$$
\begin{aligned}
& \int_{\mathcal{H}_{R} \cap\{t<\varepsilon\}} v\left|\nabla u_{R}\right|^{p-2} \nabla u_{R} \cdot \nabla \xi_{\varepsilon} \mathrm{d} x \\
& \leq C\left(\int_{\mathcal{H}_{R} \cap\{t<\varepsilon\}}\left|\nabla u_{R}\right|^{p} \mathrm{~d} x\right)^{\frac{p-1}{p}}\left(\int_{\mathcal{H}_{R} \cap\{t<\varepsilon\}}\left|\nabla \xi_{\varepsilon}\right|^{p} \mathrm{~d} x\right)^{\frac{1}{p}} .
\end{aligned}
$$

Since $\left|\nabla \xi_{\varepsilon}\right|^{p} \leq C / \varepsilon^{p},\left|\mathcal{H}_{R} \cap\{t<\varepsilon\}\right| \leq C_{R} \varepsilon^{m}$, and $m \geq p$, the second factor in the right hand side is bounded independently of $\varepsilon$. At the same time, the first factor tends to zero as $\varepsilon \rightarrow 0$ since $\left|\nabla u_{R}\right|^{p}$ is integrable in $\mathcal{H}_{R}$.

We have established the existence of a solution $u_{R}$ in $\mathcal{H}_{R}=B_{R} \cap\{s>t\}$ with $0 \leq u_{R} \leq M$. Considering the odd reflection of $u_{R}$ with respect to the Simons cone $\mathcal{C}$,

$$
u_{R}(s, t)=-u_{R}(t, s),
$$

we obtain a weak solution in $B_{R} \backslash\{0\}$. That is to say we have

$$
\int_{B_{R}}\left[\left|\nabla u_{R}\right|^{p-2} \nabla u_{R} \cdot \nabla \eta-f\left(u_{R}\right) \eta\right] \mathrm{d} x=0, \quad \text { for all } \eta \in W_{0}^{1, p}\left(B_{R} \backslash\{0\}\right) \text {. }
$$

Using the same argument as above, we can conclude that $u_{R}$ is also a solution around 0 , and hence in all of $B_{R}$. More precisely, we set $\eta=v\left(1-\xi_{\varepsilon}\right)$ in (3.5), where $v \in C_{c}^{\infty}\left(B_{R}\right)$ and $\xi_{\varepsilon}$ is identically 1 in $B_{\frac{\varepsilon}{2}}(0)$ and vanishes outside of $B_{\varepsilon}(0)$. Then (3.5) becomes

$$
\begin{aligned}
& \int_{B_{R}}\left(1-\xi_{\varepsilon}\right)\left|\nabla u_{R}\right|^{p-2} \nabla u_{R} \cdot \nabla v \mathrm{~d} x-\int_{B_{\varepsilon}} v\left|\nabla u_{R}\right|^{p-2} \nabla u_{R} \cdot \nabla \xi_{\varepsilon} \mathrm{d} x \\
& \quad=\int_{B_{R}} f\left(u_{R}\right) v\left(1-\xi_{\varepsilon}\right) \mathrm{d} x .
\end{aligned}
$$


It is clear that $\int_{B_{\varepsilon}} v\left|\nabla u_{R}\right|^{p-2} \nabla u_{R} \cdot \nabla \xi_{\varepsilon} \mathrm{d} x$ tends to zero as $\varepsilon \rightarrow 0$. Hence we conclude that $u_{R}$ is also a weak solution in $B_{R}$.

We now wish to pass to the limit in $R$ and obtain a solution in all of $R^{2 m}$. From [14], we know that for given $S>0$, we have

$$
\left|\nabla u_{R}\right| \leq C \quad \text { in } B_{S}, \quad \text { for all } R>S+2,
$$

for some constant $C$ independent of $S$ and $R$. [14] also gives a uniform $C^{1, \beta}\left(\bar{B}_{S}\right)$ bound for $u_{R}$, for some $\beta \in(0,1)$. By the Arzela-Ascoli theorem, a subsequence of $\left\{u_{R}\right\}$ converges in $C^{1}\left(\bar{B}_{S}\right)$ to a weak solution in $B_{S}$. Taking $S=1,2, \ldots$ and making a Cantor diagonal argument, we obtain a sequence $u_{R_{j}}$ converging in $C_{\text {loc }}^{1}\left(R^{2 m}\right)$ to a weak solution $u$.

By construction, we have that $u$ is a weak solution in $R^{2 m}$ depending only on $s$ and $t$, odd with respect to the Simons cone $\mathcal{C}$, with $|u| \leq M$ in $R^{2 m}$, and with $u \geq 0$ in $\{s>t\}$. Since $f \in C^{1}$ and $u \not \equiv M$, from Lemma 2.4 we have $u<M$ in $\mathcal{H}$. As a consequence, we also have $u>-M$. We claim that $u \not \equiv 0$ in $R^{2 m}$. In the light of (1.10), $u \geq 0$ and $u \not \equiv 0$ in $\mathcal{H}$, Theorem 2.3 gives $u>0$ in $\mathcal{H}$. Thus, the function $u$ has all the properties of a saddle solution as in Definition 1.2.

We show $u \not \equiv 0$. Since $u_{R}$ minimizes the energy, the result in [18] shows

$$
\int_{\mathcal{H}_{S}}\left(\frac{\left|\nabla u_{R}\right|^{p}}{p}+F\left(u_{R}\right)\right) \mathrm{d} x \leq C S^{2 m-1},
$$

where constant $C$ independent of $S$ and $R$. We now let $R=R_{j} \rightarrow \infty$ to obtain

$$
\int_{\mathcal{H}_{S}}\left(\frac{|\nabla u|^{p}}{p}+F(u)\right) \mathrm{d} x \leq C S^{2 m-1}
$$

for some constant $C$ independent of $S$. Note that this bound, after odd reflection with respect to $\mathcal{C}$, establishes the energy bound

$$
J\left(u, B_{S}\right) \leq C S^{2 m-1},
$$

which is estimate (1.12) in the statement of the theorem.

Suppose that $u \equiv 0$. Then the energy in (3.7) would read

$$
c_{m} F(0) S^{2 m}=F(0)\left|B_{S}\right|=J\left(0, B_{S}\right) \leq C S^{2 m-1} .
$$

This a contradiction for $S$ large, and thus $u \not \equiv 0$.

Finally, we establish the last statement of the theorem on stability under perturbations vanishing on the Simons cone. We assume $f$ also satisfies $\frac{f^{\prime}(w)}{f(w)} \leq$ $\frac{p-1}{w}$ in $(0, M)$. Hence $u$ weakly satisfies

$$
-\Delta_{p} u=f(u) \geq \frac{f^{\prime}(u) u}{p-1} \quad \text { in } \mathcal{H} .
$$

Namely

$$
\int_{\mathcal{H}}\left[|\nabla u|^{p-2} \nabla u \cdot \nabla \eta-\frac{f^{\prime}(u) u}{p-1} \eta\right] \mathrm{d} x \geq 0, \quad \text { for all } \eta \geq 0 \in W_{0}^{1, p}(\mathcal{H}) .
$$

In the inequality (3.8) we take $\eta=(p-1) \frac{\xi^{2}}{u} \geq 0$, where any $\xi \in C^{1}$ with compact support in $\mathcal{H}$ (and not necessarily depending only on $s$ and $t$ ), we can 
get that the value of the quadratic form $Q_{u}(\xi)$ is nonnegative. By an approximation argument, the same holds for all $\xi \in C^{1}$ with compact support in $\overline{\mathcal{H}}$ and vanishing on $\partial \mathcal{H}=\mathcal{C}$. Finally, by odd symmetry with respect to $\mathcal{C}$, the same is true for all $C^{1}$ functions $\xi$ with compact support in $R^{2 m}$ and vanishing on $\mathcal{C}$.

Finally, we prove Theorem 1.5.

Proof. Let $u$ be a bounded weak solution of $-\Delta_{p} u=f(u)$ in $R^{2 m}$ that vanishes on the Simons cone $\mathcal{C}=\{s=t\}$. We wish to show that

$$
|u(x)| \leq\left|u_{0}\left(\frac{s-t}{\sqrt{2}}\right)\right| \quad \text { in } R^{2 m}
$$

First we prove that $|u|<M$. Arguing by contradiction, assume that $|u| \geq M$ somewhere. Since $u(0)=0$, there exists a point $x_{0}$ such that $u\left(x_{0}\right)= \pm M$. Then, by Theorem 2.2 we know $u$ is constant, since $F\left(u\left(x_{0}\right)\right)=0$. On the other hand, $u=0$ on the Simons cone, we must have $u \equiv 0$. This is a contradiction.

Since $|u|<M$, now we may set

$$
v(x)=u_{0}^{-1}(u(x)),
$$

that is, $u(x)=u_{0}(v(x))$, where $u_{0}$ is the one dimensional monotone solution whose existence is given by Lemma 2.5, with $m_{1}=-M$, and $m_{2}=M$, and such that $u_{0}(0)=0$. Now, (2.4) written in terms of $v$ becomes

$$
\left|u_{0}^{\prime}\right|^{p}|\nabla v|^{p} \leq \frac{p}{p-1} F\left(u_{0}(v)\right) \quad \text { in } R^{2 m} .
$$

By $(2.8)$, recalling that $u_{0}^{\prime}(\tau)>0$, we have

$$
|\nabla v| \leq 1 \quad \text { in } R^{2 m}
$$

Finally, since $u=0$ on $\mathcal{C}$, we also have $v=0$ on $\mathcal{C}$. Given $x \in R^{2 m}$, let $x_{0} \in \mathcal{C}$ be such that $\left|x-x_{0}\right|=\operatorname{dist}(x, \mathcal{C})$. Then,

$$
|v(x)|=\left|v(x)-v\left(x_{0}\right)\right| \leq\|\nabla v\|_{L^{\infty}}\left|x-x_{0}\right| \leq\left|x-x_{0}\right|=\operatorname{dist}(x, \mathcal{C}) .
$$

By Lemma 4.2 in [6], using that $u_{0}$ is odd since $f$ is odd and that $u_{0}$ is increasing, we conclude

$$
|u(x)|=\left|u_{0}(v(x))\right|=u_{0}(|v(x)|) \leq u_{0}(\operatorname{dist}(x, \mathcal{C}))=\left|u_{0}\left(\frac{s-t}{\sqrt{2}}\right)\right|,
$$

which is the desired bound. Finally, we prove the last statement of the theorem, that is, the fact $u_{0}\left(\frac{s-t}{\sqrt{2}}\right)$ is a supersolution of $-\Delta_{p} u=f(u)$ in the set $\mathcal{H}=\{s>t\}$. First, by direct computation using Eq. (3.2) in $(s, t)$ variables for $t>0$ gives that the function is a supersolution in $\{s>t>0\}$. By a capacity argument, we can prove that $u_{0}\left(\frac{s-t}{\sqrt{2}}\right)$ is a supersolution in $\mathcal{H}$. 


\section{Acknowledgments}

This research is partially supported by an Oversea Cooperation Fund of National Science Foundation of China. The first author is partially supported by NSFC, No. 10971057. The third author is supported by the National Science Funds of China (10971061). The first and second authors would like to thank their advisor Professor Changfeng Gui for his patient guidance. The authors would also like to thank the anonymous referee for several very helpful suggestions.

\section{References}

[1] Alama, S., Bronsard, L., Gui, C.: Stationary layered solutions in $R^{2}$ for an AllenCahn system with multiple well potential. Calc. Var. Partial Differ. Equ. 5, 359-390 (1997)

[2] Alberti, G., Ambrosio, L., Cabré, X.: On a long-standing conjecture of E. De Giorgi: symmetry in 3D for general nonlinearities and a local minimality property. Acta Appl. Math. 65(1-3), 9-33 (2001)

[3] Alessio, F., Calamai, A., Montecchiari, P.: Saddle-type solutions for a class of semilinear elliptic equations. Adv. Differ. Equ. 12, 361-380 (2007)

[4] Ambrosio, L., Cabré, X.: Entire solutions of semilinear elliptic equations in $R^{3}$ and a conjecture of De Giorgi. J. Am. Math. Soc. 13, 725-739 (2000)

[5] Berestycki, H., Caffarelli, L., Nirenberg, L.: Further qualitative properties for elliptic equations in unbounded domains. Ann. Scuola Norm. Sup. Pisa Cl. Sci. (4) 25, 69-84 (1997)

[6] Cabré, X., Terra, J.: Saddle solutions of bistable diffusion equations in all of $R^{2 m}$. J. Eur. Math. Soc. (JEMS) 11(4), 819-843 (2009, to appear)

[7] Cabré, X., Terra, J.: Qualitative properties of saddle-shaped solutions to bistable diffusion equations, preprint

[8] Caffarelli, L., Cordoba, A.: Uniform convergence of a singular perturbation problem. Comm. Pure Appl. Math. 48(1), 1-12 (1995)

[9] Caffarelli, L., Garofalo, N., Segàla, F.: A gradient bound for entire solutions of quasilinear equations and its consequences. Comm. Pure Appl. Math. 47, 14571473 (1994)

[10] Dang, H., Fife, P.C., Peletier, L.A.: Saddle solutions of the bistable diffusion equation. Z. Angew Math. Phys. 43, 984-998 (1992)

[11] Danielli, D., Garofalo, N.: Properties of entire solutions of non-uniformly elliptic equations arising in geometry and in phase transitions. Calc. Var. Partial Differ. Equ. 15(4), 451-491 (2002) 
[12] De Giorgi, E.: Convergence problems for functionals and operators. In: Proceedings of the Internartional Meeting on Recent Methods in Nonlinear Analysis (Rome, 1978), Pitagora, Bologna, pp. 131-188 (1979)

[13] del Pino, M., Kowalczyk, M., Wei, J.: On De Giorgi conjecture in dimension $N \geq 9$, Preprint

[14] Dibenedetto, E.: $C^{1+\alpha}$ local regularity of weak solutions of degenerate elliptic equations. Nonlin. Anal. T.M.A. 7(8), 827-850 (1983)

[15] Ghoussoub, N., Gui, C.: On a conjecture of De Giorgi and some related problems. Math. Ann. 311, 481-491 (1998)

[16] Ghoussoub, N., Gui, C.: On De Giorgi's conjecture in dimensions 4 and 5. Ann. Math.(2) 157(1), 313-334 (2003)

[17] Jerison, D., Monneau, R.: Towards a counter-example to a conjecture of De Giorgi in high dimensions. Ann. Mat. Pura Appl. 183, 439-467 (2004)

[18] Petrosyan, A., Valdinoci, E.: Density estimates for a degenerate/singular phasetransition model. SIAM J. Math. Anal. 36(4), 1057-1079 (2005)

[19] Savin, O.: Regularity of flat level sets in phase transitions. Ann. Math. (to appear)

[20] Schatzman, M.: On the stability of the saddle solution of Allen-Cahn's equation. Proc. R. Soc. Edinb. Sect. A 125, 1241-1275 (1995)

[21] Sciunzi, B., Valdinoci, E.: Mean Curvature properties for p-Laplace phase transitions. J. Eur. Math. Soc. (JEMS) 7(3), 319-359 (2005)

[22] Valdinoci, E., Sciunzi, B., Savin, O.: Flat level set regularity of $p$-Laplace phase transitions. Mem. Amer. Math. Soc. 182(858), vi+144pp (2006)

[23] Vazquez, J.L.: A strong maximum principle for some quasilinear elliptic equations. Appl. Math. Optim., pp. 191-202 (1984)

Z. Du

College of Mathematics and Econometrics,

Hunan University,

Changsha 410082,

China

e-mail: zhuorandu@yahoo.com.cn

\section{Z. Zhou}

Department of Mathematics and Physics,

Xiamen University of Technology,

Xiamen 361024,

China

e-mail: zzzzhhhoou@yahoo.com.cn 
B. Lai

Institute of Contemporary Mathematics,

Henan University School of Mathematics and Information Science,

Henan University, Kaifeng 475004,

China

e-mail: laibaishun@henu.edu.cn

Received: 27 January 2010.

Revised: 28 July 2010.

Accepted: 02 September 2010. 